\title{
Research on Thermal Decomposition of Double-base Propellant
}

\author{
WANG Heng-chuang, CHEN Ming-hua, CHEN Yong-kang, GE Qiang, WANG Shao-guang \\ Ordnance Engineering College, \\ Shijiazhuang 050000, China \\ e-mail: wanghengchuang@163.com
}

\begin{abstract}
The importance of the quantum chemical method and thermal decomposition kinetics on double-base propellant was reviewed. The main analysis methods and theory mode of thermal decomposition kinetics were enumerated and analyzed. It is significant to put quantum chemical method and thermal decomposition kinetics together on thermal decomposition of energetic materials especially double-base propellant. That provide science basis for safe function assesses of the propellant. It provides science basis for the forecast of service life and the evaluation of security.
\end{abstract}

Keywords-double-base propellant; quantum chemical; thermal decomposition kinetics; theory mode; forecast of life

\section{INTRODUCTION}

The thermal analysis phenomenon that discovered at the earliest stage was hot to lose heavy, which is observed firstly when 1786 studied a porcelain and ceramics to glue soil by British Edgwood, but it is still in the 50's in 20th century that thermal analysis technique really meaning establish and develop [1-2]. The thermal analysis dynamics was applied theoretically inquire into physics variety and the mechanism of the chemical reaction, and building up the relation of process progress, time and temperature on the application, that can be used for the service life that predicts material and evaluate the risk of containing the ability material and provide a storage condition. The characteristics of thermal decomposition of energetic materials are explained by thermal analysis technique [3]. At the same time, applying quantum chemistry into the hot decomposition of energetic materials especially the double-base propellant, and by way of quantum chemistry calculation method to set up the hot decomposition mechanism that double-base propellant, and used with hot analysis method, that will consumedly raise research efficiency.

\section{Method of Quantum ChemistRy}

In recent years, the calculation method of the quantum chemistry and the rapid development of the calculator operation speed, make the quantum chemistry get an extensive application in the research [4-8] on energetic materials.

Make use of a quantum chemistry research method, according to propellant molecules system of size, to choice accommodation of DFT calculation method, to shoot digest fiber and nitroglycerine ground state molecules within propellant of balance several the structure carry on excellent turn, and set up heat to resolve reaction mechanism. It computes some thermodynamics data including a reaction ability, enthalpy and freedom energy thermodynamics data etc. The above work exists a crux and includes the calculation of transferring the Tai and certainly ability setting up of noodles. The velocity constant computes and stirs up Tai of the balance are several what structure of calculation and melting agent turn the influence of effect.

Wang Xue-jie [9] adopted the method of TG and DSC measures PZFX is in nitrogen atmosphere and empty atmosphere in of hot resolve process, made use of the quantum chemistry GAMESS software to compute the key class of the molecules of PZFX to predict PZFX hot decomposition mechanism.

Xue Yun-na [10] adopted Gaussian 98 medium DFTB3 LYP method at the 6-31 Gs(d, p) radical set level bottom to 3,4-the double(3' -nitrics radicle benzenes-1 ' -radical) oxidize Mao, we carry on all of structure excellent turn and Wiberg key class calculation, and adopt TG-DSC-FITR-MS which is an analyze method as to research on the hot decomposition mechanism. 
Zhang Xing-gao outlined the compound, hot decomposition and application prospects of high nitrogen synthesizing. Making use of the research method of quantum chemistry to high nitrogen synthesizing a theory calculation, getting the calculation of MNDO calculate way to the born Han of high nitrification thing is more accurate.

Liu Zhi-yong [11] stable geometry of isopropyl nitrate, a kind of propellant, was obtained from energy optimization calculation by quantum chemistry method. The static property was analyzed on this basis the theory at firework character ability of research realm, especially the estimate resolves the mechanism have with reaction path aspect to know a function greatly.

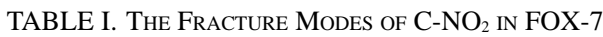

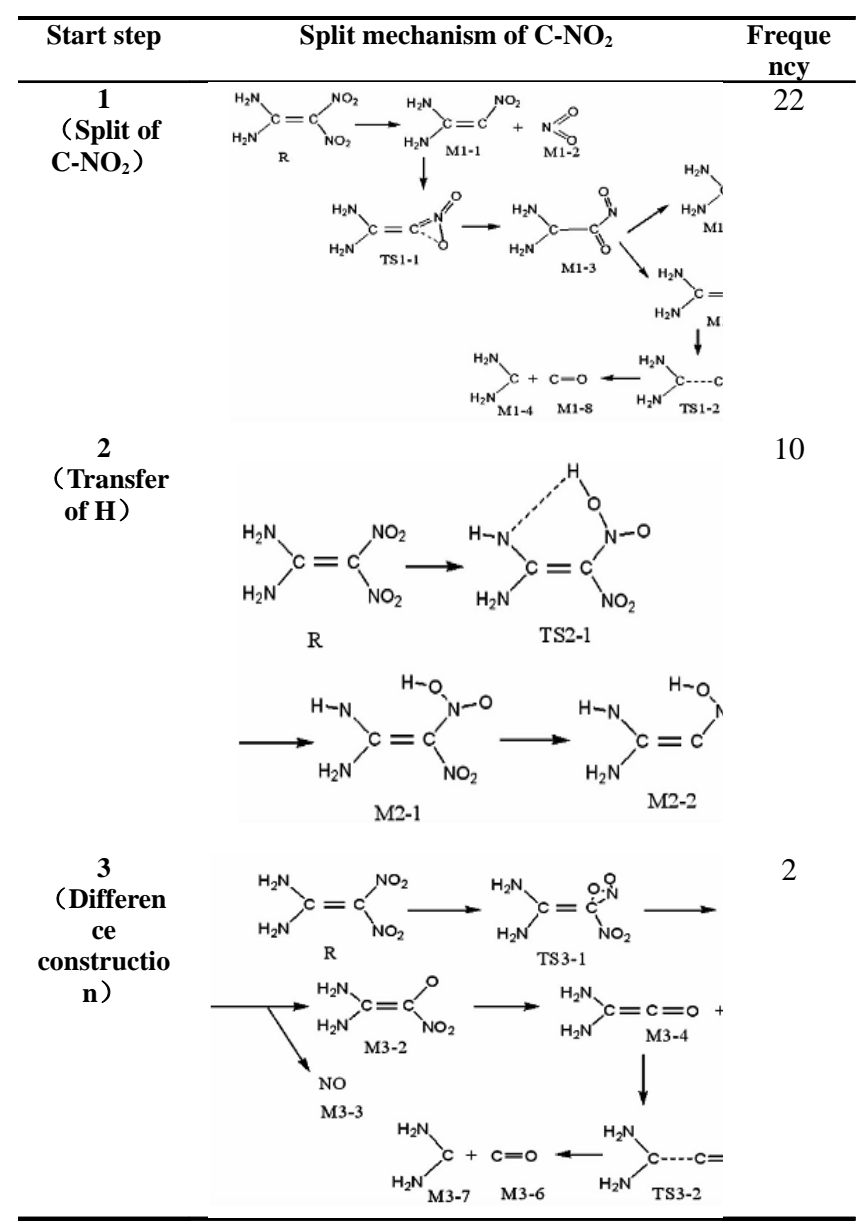

Zong He-hou [12] took FOX-7 as an example to introduce that adopting the method of the density is suffused with a letter to study FOX-7 according to the quantum chemistry theory and it two kinds of molecules that can divide a different construction reach type and hot decomposition together. Table I listed splitting of C-
$\mathrm{NO}_{2}$ keys, hydrogen to transfer and the difference reach to turn to appear the reaction of most frequency in the reaction.

Through above discussion, I get some questions need to be consummate. (1) The quantum chemistry method needs further exaltation accuracy and lower calculation to consume. (2) Combining quantum chemistry with molecules dynamics can make of hot decomposition data credibility higher, (3) Development of quantum chemistry method provide chemical reaction dynamics method with vast apply prospect, but it is limited that the chemical reaction dynamics uses in the research broken key reaction.

\section{Dynamics Method}

At the hot decomposition of the energetic material, resolve calories and quality data the loss usually is the total pack of process of decomposition, only depend it is can hardly for hot analysis income data to acquire reaction mechanism, for accurate study it hot resolve process, have to the outcome carry on analytical the in common use method has the adoption TG-DSC-FTI R and TG-DSC-MS for energetic material of molecules decomposition the step and mechanism research provide a great deal of data [13].

\section{a) Theory Model}

The heat resolves a velocity equation:

$$
\frac{d a}{d t}=K(\mathrm{~T}) f(\mathrm{a})
$$

Among that, $a$ is conversion rate, $K(T)$ is reaction kinetics constant, $f(a)$ is responds mechanism function.

For push forward the hot decomposition of, assumption reaction kinetics constant IC(T) with respond that the relation of temperature $\mathrm{T}$ can be meant with the Arrehenius equation:

$$
K(\mathrm{~T})=A \exp \left(-\frac{E}{R_{\mu} T}\right)
$$

Respond mechanism function adopts a type:

$$
f(a)=(1-a)^{n}
$$

Put (2.2) 、 (2.3) in (2.1), tidy up:

$$
\ln \frac{d a}{d a t}=\ln A-\frac{E}{R_{\mu} T}+n \ln (1-\mathrm{a})
$$

For the DSC diagram, often adopt the type compute a conversion rate $a$ : 


$$
a=\frac{\int_{T_{1}}^{T} \dot{H} d T}{\beta m Q}
$$

Then

$$
\frac{d a}{d t}=\frac{\dot{H}}{m Q}
$$

Where, $m$ is sample quality, $Q$ is number of hot effect, $T_{i}$ is the start temperature of resolve reaction [14].

\section{b) Kinetic Analysis Method}

In addition, some comment dynamics methods contain method of Zivkovic [15], method of Doyle and method of Agrawal [16], method of Freeman-Carroll [17], method of Friedman [18], method of Starink [19], method of Anderson-Freeman [20], method of Popescu [21] and method of Criado standard curve [22] and method of method of Coats-Redfern $[23,24]$ etc.s. Table II medium listed this a few dynamics analysis methods.

\begin{tabular}{|c|c|}
\hline method & dynamics equation \\
\hline $\begin{array}{l}\text { Method of } \\
\text { Zivkovic }\end{array}$ & $\ln \{\ln [1 /(1-\alpha)] / \mathrm{t}\}=\ln \mathrm{A}-\mathrm{E} / \mathrm{RT}$ \\
\hline Method of Doyle & $\ln \beta=\ln [A E / R G(\alpha)]-2 \ln (\mathrm{E} / \mathrm{RT})-\mathrm{I}$ \\
\hline $\begin{array}{l}\text { Method of } \\
\text { Agrawal }\end{array}$ & $\begin{array}{l}\ln \left[G(\alpha) / \mathrm{T}^{2}\right]=\ln \{[A R(1-2 \mathrm{RT} / \mathrm{E}) / \beta \mathrm{E}] / \\
\left.\left[1-5(\mathrm{RT} / \mathrm{E})^{2}\right]\right\}-E / R T\end{array}$ \\
\hline $\begin{array}{c}\text { Method of } \\
\text { Freeman- } \\
\text { Carroll }\end{array}$ & $\begin{array}{l}\Delta \lg (\mathrm{d} \alpha / \mathrm{dt}) / \Delta \lg (1-\alpha)=-\mathrm{E}[\Delta(1 / \mathrm{T}) / \\
\Delta \lg (1-\alpha)] / 4.575+\mathrm{n}\end{array}$ \\
\hline $\begin{array}{l}\text { Method of } \\
\text { Friedman }\end{array}$ & $\ln [\beta d \alpha / \mathrm{dT}]=\ln [\mathrm{A} f(\alpha)]-\mathrm{E} / \mathrm{RT}$ \\
\hline $\begin{array}{l}\text { Method of } \\
\text { Starink }\end{array}$ & $\ln \left[\beta / \mathrm{T}^{1.8}\right]=\mathrm{C}_{s}-10037 E / R T$ \\
\hline $\begin{array}{l}\text { Method of } \\
\text { Anderson- } \\
\text { Freeman }\end{array}$ & $\begin{array}{l}\Delta \lg (d \alpha / d T)=-\mathrm{E}[\Delta(1 / \mathrm{T})] / 2.303 \mathrm{R}+ \\
\mathrm{n}[\Delta \lg (1-\alpha)]\end{array}$ \\
\hline
\end{tabular}

TABLE II. Kinetic AnAlysis Method

Note: $t$ for time(s); $C_{s}$ for the constant; $f(a)$ for the differential calculus form of mechanism function; $n$ for respond series.

\section{c) Forecast of Life}

It is easy for propellant to be influence by external environment, that the stability is worse of the propellant. The stabilize function can influence the safety of storage of propellant directly. Thus, it is an important military meaning and economic meaning to do some research on stabilize of propellant and forecast the life of safely storage.

Dakin [25] put forward and apply experiment to prove that the material a number to present line sexual behavior:

$$
\lg \tau=a / T+b
$$

Where, $T$ is temperature of thermodynamics, $a=E_{\alpha} / 2303 R, b$ is a constant that is connect with reaction series $n$ and before factor refers $A$. The life equation can be established according to activation energy $E_{a}$ and the time of the enactment that experiment get lose heavy for leading conversion.

\section{CONCLUSION}

The development of quantum chemistry provides vast visual fields for chemical reaction dynamics method, it can convenient get the data that the experiment can't get, it combines with molecules dynamics to make thermal decomposition data more credibility, more enriching information is one of the important development directions of theory research hot decomposition aftertime. The hot decomposition dynamics developed an important function in the aspects of solving to contain ability the dynamics parameter of the material predicting reaction mechanism, hot analysis technique oneself of perfect and experiment and calculator technique are gradually perfect, that make quantum chemistry method and heat analytical technique of allied use at energetic material especially hot decomposition mechanism developed of propellant take an important function in the research, the quantum chemistry method needed to depend on the test of verify accuracy, experiment provided a calculating basis for the quantum chemistry method, it get addition and verify mutually with dynamics heat analysis of double-base propellant dynamics heat analysis to use.

\section{REFERENCE}

[1] J.H. Flynn, “Thermal analysis kinetics- past, present and future,” Thermochimica Acta, vol. 203 (1), pp. 519-526, 1992.

[2] S. Vyazovkin, and C.A. Wight, "Isothermal and non- isothermal kinetics of thermally stimulated reactions of solids,” Intemational Reviews in Physical Chemistry, vol. 17, pp. 407-433, 1998.

[3] H.R. Zu, and G.S. Li, “Hot Analysis Dynamics,” Beijing: Science 
publisher, 2008.

[4] R.G. Parr, and W. Yang, Density-Functional, "Theory of Atoms and Molecules,” Oxford Univ Press, 1989.

[5] J.K. Labanowski, and J.W. Andzelm, "Density Functional Methods in Chemistry,” New York: Springer-verlag, 1991.

[6] P. Hohenberg, and W. Kohn, “Inhomogeneous electron gas,” Phys Rev B, 1964, pp. 136-864.

[7] W. Kohn, and L.J. Sham, "Self-consistent equations including exchange and correlation effects,” Phys Rev A, vol. 140, pp. 11-33, 1965.

[8] D.R. Salahub, and M.C. Zerner, "The challenge of $d$ and $f$ electrons,” ACS Symposium Series, Washington D C: ACS, 1989.

[9] W.X. Jie, "Mechanism and Kinetics of Thermal Decomposition of Pazufloxacin Mesilate,” Journal of Analytical Science, vol. 27, 2011.

[10]X.Y. Na, and Y.J. Ming, "Thermal Decomposition Mechanism and Quantum Chemistry Study on 3, 4- Bis (3'-nitrophenyl-1'-yl) furoxan,” Chinese Journal of Energetic Materials, vol. 19, pp.1318, 2011.

[11]L.Z. Yong, and Y. Hua, “Analysis on the Decomposition Mechanism of a Kind of Propellant by Quantum Chemistry Method,” Initiators \& Pyrotechnics, 2007.

[12]Z.H. Hou, "Theoretical study on the thermal decomposition of FOX-7 and crystal characteristics,” Mianyang: China Academy of Engineering Physics, 2007.

[13]L.Z. Ru, "Hot Analysis of Energetic Materials," Beijing: Defense industry publisher, 2008.

[14]S. Hui, "Study on Thermal Analysis, Ignition and Combustion Characteristics of AP/HTPB base bleed propellant," Nanjing University of Science \& Technology, 2013.

[15]Z.D. Zivkovic, and B. Dobovisek, "Determination of reaction kinetics based on a part of a differential thermal analysis or thermo gravimetric curves,” Thermochimica Acta, vol. 32, pp. 205- 211, 1979.

[16]R.K. Agrawal, “A new equation for modeling no isothermal reactions,” Chemistry Analysis, vol. 32, pp. 149-156, 1987

[17]E.S. Freeman, and B. Carroll, "The application of thermo analytical techniques to reaction kinetics:the thermogravimetric evaluation of the kinetics of the decomposition of calcium oxalate monohydrate,” The Journal of Physica Chemistry, vol. 62, pp. 394397, 1958.

[18]H.L. Friedman, "Kinetics of thermal degradation of charforming plastics from thermograximetry. Application to a phenolic plastic," Journal of Polymer Science, Part C, vol. 6, pp. 183-195, 1964.

[19]M.J. Starink, "A new method for the derivation of activation analysis from experiments performed at constant heating rate," Thermochimica Acta, vol. 288, pp. 97-104, 1996.

[20]D.A. Anderson, and E.S Freeman, "The kinetics of the thermal degradation of polystyrene and polyethylene,” Journal of Polymer Science, vol. 54, pp. 253-260, 1961.

[21]C. Popescu, "Integral method to analyze the kinetics heterogeneous reactions under non-isothermal condition a variant on the OzawaFlynn-Wall method,” Thermochimica Acta, vol. 285, pp. 309-323, 1996.

[22]J.M. Criado, "Kinetic analysis of DTA data from master curves," Thermochimica Acta, vol. 24, pp. 186-198, 1978.

[23]A.W. Coats, and J.P. Redfern, "Kinetic parameters from thermogravimetric data," Nature, 1964, pp. 68-69.

[24]N.B. Ke, Y.Z. Quan and L. Rong, "A study on the denitration kinetics of highly nitrated nitrocellu-lose," Chinese Journal of Explosives \& Propellant, vol. 23, pp.65-67, 2000.

[25]T.W. Dakin, "Electrical Insulation Deterioration Treated as a Chemical Rate Phenomena," AIEE Transactions, Part I (Communication and Electronics), vol. 67, pp. 11, 1948. 